\title{
Editorial: Optogenetics: An Emerging Approach in Cardiac Electrophysiology
}

\author{
Christopher L.-H. Huang ${ }^{1,2 *}$, Emily A. Ferenczi ${ }^{3}$ and Ming Lei ${ }^{2,4 *}$ \\ ${ }^{1}$ Physiological Laboratory and Department of Biochemistry, University of Cambridge, Cambridge, United Kingdom, ${ }^{2}$ Key \\ Laboratory of Medical Electrophysiology of the Ministry of Education and Institute of Cardiovascular Research, Southwest \\ Medical University, Luzhou, China, ${ }^{3}$ Department of Neurology, Harvard Medical School, Massachusetts General Hospital and \\ Brigham and Women's Hospital, Boston, MA, United States, ${ }^{4}$ Department of Pharmacology, University of Oxford, Oxford, \\ United Kingdom
}

Keywords: optogenetics, cardiac electrophysiology, cardiac arrhythmias, clinical translation, opsins

\section{Editorial on the Research Topic}

\section{OPEN ACCESS}

Edited and reviewed by: Ruben Coronel,

University of Amsterdam, Netherlands

*Correspondence:

Christopher L.-H. Huang clh11@cam.ac.uk

Ming Lei

ming.lei@pharm.ox.ac.uk

Specialty section:

This article was submitted to

Cardiac Electrophysiology,

a section of the journal

Frontiers in Physiology

Received: 16 March 2020

Accepted: 06 April 2020

Published: 28 April 2020

Citation:

Huang CL-H, Ferenczi EA and Lei M (2020) Editorial: Optogenetics: An

Emerging Approach in Cardiac

Electrophysiology.

Front. Physiol. 11:414.

doi: 10.3389/fphys.2020.00414

\section{Optogenetics: An Emerging Approach in Cardiac Electrophysiology}

Optogenetic techniques modulate excitable membranes through activation of photosensitive opsin proteins functioning as light-gated channels, transporters or receptors. Of the known, prokaryotic, algal or fungal, type I, and animal, type II, opsins, the type I opsins have been the most frequently used experimentally since they were first to be expressed in mammalian neurons in the early 2000 s. Optogenetic approaches circumvent disadvantages of traditional, direct electrical stimulation, methods, such as tissue toxicity, and enhance cellular and spatial specificity of stimulus effects through genetic or developmental targeting.

These methods initially found neuroscience applications and these ranged widely from use in in vitro models to behaving animals, and in invertebrate through to mammalian systems (Boyden et al., 2005). They have more recently proved relevant to other excitable, particularly cardiac, tissues, in the latter case, particularly in fundamental scientific and translational studies of cardiac arrhythmogenesis (Bruegmann et al., 2016). Arrhythmias are the result of disruption of the normally orderly electrical excitation sequence initiating co-ordinated and effective atrial or ventricular contraction. They account for $\sim 3.7$ million human deaths/year worldwide (Kuriachan et al., 2017). Their clinical management has often lagged progress in many other cardiological areas. This likely reflects our currently limited understanding of the physiological mechanisms that underly their initiation, maintenance or propagation (Huang, 2017; Lei et al., 2018; Huang et al., 2020).

Cardiac optogenetic approaches offer unprecedented opportunities and new exciting possibilities to resolving and intervening in such normal and diseased function. Optogenetic sensors can be incorporated into specific motifs and targeted at specific, tissue and cell types, and subcellular domains, and applied at high spatial and temporal resolution. This Research Topic 
collects articles focusing on optogenetic applications in cardiac electrophysiology. They particularly consider their ability to modify membrane voltage, intracellular $\mathrm{Ca}^{2+}$ and cellular signaling, and their potential for use in cardiac pacing, cardioversion, cell communication, in arrhythmia research (Boyle et al., 2018). Pioneering contributors in this cardiac field summarize underlying concepts, existing optogenetic tools and approaches, discussing their suitability for cardiac experimental studies and interventions. They then review examples of applications and situations where optogenetics have yielded new insights into and potential interventional approaches for cardiac physiology and cardiac disease.

Two background articles explore available optogenetic approaches, opsin proteins and their different ion conductances, kinetics, light sensitivity, and activation spectra, both in general, and bearing on cardiac applications (Ferenczi et al.; O'Shea et al.). These include the physical and practical background, and choices of opsin tools appropriate to the cellular physiology and experimental system in question (Deisseroth, 2015). Cardiac expression of the chosen optogenetic tool is variously achieved by coupling of "spark cells" at the single-cell level, construction of transgenic mice or in vivo introduction of adenoviral expression systems at the systems level. Adeno-associated viruses (AAVs) offer advantages in long-term, cardiac-specific gene expression. An article exploring optimal AAV serotypes $(1,6$, or 9$)$ reports preferential efficiency of AAV1/6 in vitro and AAV9 in vivo for cardiac optogenetic delivery (Ambrosi et al.). Optogenetic stimulation either activates or silences cellular excitation through membrane depolarization or hyperpolarization. Depolarizing, activated, cation-conducting channelrhodopsins (ChR) trigger cardiomyocyte action potentials; hyperpolarization by $\mathrm{Cl}^{-}$ pumps suppresses activity (Arrenberg et al., 2010). The activated photosensitive $\mathrm{Cl}^{-}$channel, Guillardia theta ChR (GtACR1) silences neuronal action potential generation: microelectrode and in vivo optical whole heart recordings demonstrate depolarized cells as expected from the cardiac $\mathrm{Cl}^{-}$Nernst potential, inhibiting re-excitation (Kopton et al.).

Optogenetic techniques promise to deliver unique physiological insights unobtainable from established procedures. At the cellular level, it could demonstrate, localize, and characterize electrical and $\mathrm{Ca}^{2+}$ transient activity in particular phenylethanolamine-N-methyl transferase (Pnmt)-derived cardiomyocyte subpopulations and their roles in localized adrenergic signaling (Fan et al.). At the whole heart level, optogenetic methods permitted temporally precise, acute, neuron-specific, parasympathetic stimulation in mice expressing ChR2 in their peripheral cholinergic neurons. This elicited sudden, dramatic but reversible heart rate reductions and atrioventricular conduction delays (Moreno et al.).

Optogenetic techniques can target and stably deliver perturbing light stimuli to specific cell types at the cellular, organ or whole organism levels, in different cardiac, sino-atrial nodal, atrial, ventricular regions, or the conducting tissues within or between them, permitting focussed study of the entire range of clinically important arrhythmic conditions. Thus, protocols combining optogenetic stimulation with dual cytosolic $\mathrm{Ca}^{2+}$ membrane voltage optical mapping studied membrane potential, intracellular $\mathrm{Ca}^{2+}$ transients and pacemaker activity in a murine sino-atrial preparation (Dong et al.). Use of pseudo twodimensional murine ventricular tissue slices profiled transmural and regional voltage and $\mathrm{Ca}^{2+}$ signaling gradients under stress produced by high frequency pacing, $\beta$-adrenergic stimulation or pathological, including hypertrophic, conditions (He et al.). We also include one report where the genetically-encoded sensor for altered cellular glutathione redox status, Grx1-roGFP2, provided readouts of cellular reactive oxygen species (Trautsch et al.). The latter modulate ryanodine receptor-mediated sarcoplasmic reticular $\mathrm{Ca}^{2+}$ release (Prosser et al., 2011) and phospholamban phosphorylation-regulated diastolic relaxation (Scotcher et al., 2016).

We complete the Research Topic by considering translational exploitations of optogenetic methods in clinical cardiology. These could achieve non-damaging optical control of excitation with high temporal and spatial resolution for sino-atrial pacemaking, or atrial or ventricular cardioversion. In contrast, normally used electrical shocks do not fully control the spatial extent and level of their resulting membrane potential changes. One article summarizes recent progress on optogenetic defibrillation and cardioversion, their underlying mechanisms and potential patient populations that might thus benefit (Sasse et al.). In channelrhodopsin-2 (ChR2) transgenic mice, a single 10$1,000 \mathrm{~ms}$ pulse at light intensities $<1.10 \mathrm{~mW} \mathrm{~mm}^{-2}$ sufficed to cardiovert fibrillating hearts into sinus rhythm. Optical mapping attributed this to both annihilating, excitation wave, collisions and propagation perturbations (Quiñonez Uribe et al.). Epicardial illumination terminated ventricular arrhythmias in explanted, hypokalaemic hearts expressing the light-driven proton pump ArchT, perfused with the $\mathrm{K}_{\mathrm{ATP}}$ opener pinacidil. This was accompanied by hyperpolarization, more rapid action potential upstrokes but reduced conduction velocity implicating an enhanced electrical sink in terminating the arrhythmia. Such novel insights could improve our mechanistic understanding and treatment strategies for arrhythmia termination (Funken et al.).

\section{AUTHOR CONTRIBUTIONS}

EF surveyed and summarized all the contributions in this Research Topic and $\mathrm{ML}$ revised these, permitting $\mathrm{CH}$ to formulate the narrative underlying this Research Topic.

\section{FUNDING}

This work was funded by the Medical Research Council, UK (MR/M001288/1, CLHH; G10002647, G1002082, ML), Wellcome Trust, UK (105727/Z/14/Z,CLHH), British Heart Foundation, UK (BHF) (PG/19/59/34582, PG/14/79/31102, and PG/15/12/31280, and BHF Center for Research Excellence [CRE] at Cambridge, CLHH; PG/14/80/31106, PG/16/67/32340, PG/12/21/29473, PG/11/59/29004, and BHF CRE at Oxford, ML), National Natural Science Foundation of China (31171085, ML). 


\section{REFERENCES}

Arrenberg, A. B., Stainier, D. Y. R., Baier, H., and Huisken, S. E. (2010). Optogenetic control of cardiac function. Science 330, 971-974. doi: 10.1126/science.1195929

Boyden, E. S., Zhang, F., Bamberg, E., Nagel, G., and Deisseroth, K. (2005). Millisecond-timescale, genetically targeted optical control of neural activity. Nat. Neurosci. 8, 1263-1268. doi: 10.1038/nn1525

Boyle, P. M., Karathanos, T. V., and Trayanova, N. A. (2018). Cardiac Optogenetics 2018. JACC Clin. Electrophysiol. 4, 155-167. doi: 10.1016/j.jacep.2017.12.006

Bruegmann, T., Boyle, P. M., Vogt, C. C., Karathanos, T. V., Arevalo, H. J., Fleischmann, B. K., et al. (2016). Optogenetic defibrillation terminates ventricular arrhythmia in mouse hearts and human simulations. J. Clin. Invest. 126, 3894-3904. doi: 10.1172/JCI88950

Deisseroth, K. (2015). Optogenetics: 10 years of microbial opsins in neuroscience. Nat. Neurosci. 18, 1213-1225. doi: 10.1038/nn.4091

Huang, C. L.-H. (2017). Murine electrophysiological models of cardiac arrhythmogenesis. Physiol. Rev. 97, 283-409.doi: 10.1152/physrev.00007.2016

Huang, C. L.-H., Wu, L., Jeevaratnam, K., and Lei, M. (2020). Update on antiarrhythmic drug pharmacology. J Cardiovasc Electrophysiol 31, 579-592. doi: $10.1111 /$ jce. 14347

Kuriachan, V., Sumner, G., and Mitchell, L. (2017). Sudden cardiac death. Curr. Probl. Cardiol. 40, 133-200. doi: 10.1016/j.cpcardiol.2015.01.002
Lei, M., Wu, L., Terrar, D. A., and Huang, C. L. H. (2018). Modernized classification of cardiac antiarrhythmic drugs. Circulation 138, 1879-1896. doi: 10.1161/CIRCULATIONAHA.118.0 35455

Prosser, B. L., Ward, C. W., and Lederer, W. J. (2011). X-ROS signaling: Rapid mechano-chemo transduction in heart. Science 333, 1440-1445. doi: $10.1126 /$ science. 1202768

Scotcher, J., Prysyazhna, O., Boguslavskyi, A., Kistamas, K., Hadgraft, N., Martin, E. D., et al. (2016). Disulfide-activated protein kinase G I $\alpha$ regulates cardiac diastolic relaxation and fine-tunes the FrankStarling response. Nat. Commun. 333, 1440-1445. doi: 10.1038/ncomms 13187

Conflict of Interest: The authors declare that the research was conducted in the absence of any commercial or financial relationships that could be construed as a potential conflict of interest.

Copyright (C) 2020 Huang, Ferenczi and Lei. This is an open-access article distributed under the terms of the Creative Commons Attribution License (CC BY). The use, distribution or reproduction in other forums is permitted, provided the original author(s) and the copyright owner(s) are credited and that the original publication in this journal is cited, in accordance with accepted academic practice. No use, distribution or reproduction is permitted which does not comply with these terms. 